\title{
Actividades de Cálculo Diferencial con Computadora: Estudio de Habilidades Matemáticas Desarrolladas
}

\begin{tabular}{|c|c|c|c|}
\hline Marcela Falsetti & Adriana Favieri & Roxana Scorzo & Betina Williner \\
\hline marcelacristinaf@yahoo.com.ar & adriana.favieri@gmail.com & rscorzo@yahoo.com.ar & bwilliner@hotmail.com \\
\hline U. Nacional de La Matanza & U. Nacional de La Matanza & U. Nacional de La Matanza & U.Nacional de La Matanza \\
\hline Argentina & $\begin{array}{c}\text { Argentina } \\
\text { Recibido: } 10 \text { Octubre } 2011\end{array}$ & $\begin{array}{c}\text { Argentina } \\
\text { Aceptado: } 22 \text { Diciembre } 2012\end{array}$ & Argentina \\
\hline
\end{tabular}

Resumen. El presente artículo reporta un estudio transeccional descriptivo sobre el desarrollo de habilidades matemáticas así como la relación de éstas con actividades matemáticas y contenidos específicos. Analizamos producciones escritas de estudiantes de carreras de ingeniería que realizan su primer curso de Cálculo Diferencial. Para esta experiencia han trabajado en un taller usando el software Mathematica ${ }^{\circledR}$. Describimos los criterios considerados para la clasificación de las actividades y habilidades, los instrumentos para la evaluación y el procesamiento de los datos. En las conclusiones establecemos relaciones entre tipos de actividades y habilidades promovidas y nos referimos al rol del software en la enseñanza y en el aprendizaje de la introducción al Cálculo Diferencial. Finalmente mediante el análisis estadístico descriptivo y el de componentes principales reforzamos la hipótesis de que una habilidad debe medirse en estrecha dependencia con el contenido y la tarea realizada.

Palabras clave: Habilidades matemáticas, diseño de actividades, software matemático de cálculo simbólico y numérico, cálculo diferencial, análisis estadístico por componentes principales.

Abstract. This paper reports a transactional descriptive study on math skills, as part of mathematical competence, and its relationship with math activities and specific content. We analyze written productions of students, from Engineering careers, of the their first-Calculus course. For this experience, they have worked in a workshop using Mathematica ®software. We describe here the criteria used for the classification of activities and skills, the tools for evaluation and processing of the data. In the conclusions we establish relationships between types of activities and skills promoted and we refer to the role of software in teaching and learning in the introductory Differential Calculus course. Finally through descriptive statistical analysis and principal components analysis we also reinforce the hypothesis that a skill should be measured in close dependence on the content and the task.

KeyWords: Math Skills, design activities, mathematical calculus software, Differential Calculus, principal components analysis.

\subsection{Introducción}

En nuestro país, Argentina, las nuevas tendencias para la formación de ingenieros hacen particular hincapié en las competencias (CONFEDI, 2007), lo cual incorpora un nuevo interés formativo, ya que no solo interesa el contenido, la cantidad y la calidad de información, sino también el saber hacer y desempeñarse, la capacidad de evocar lo 
aprendido, integrarlo con otros conocimientos y aplicarlo a nuevos contextos. Según Lasnier (2000) "una competencia es un desempeñarse complejo, resultante de la integración, de la movilización y de la disposición de un conjunto de capacidades y habilidades (de orden cognitivo, afectivo, psicomotor o social), y de conocimientos (conocimientos declarativos) utilizados de manera eficaz, en situaciones que tienen un carácter común." (p.32)

Esta definición nos señala que en la formación de competencias debemos tener en cuenta las habilidades, las cuales se refieren al conocimiento en acción, al saber hacer. Preferimos abocarnos al estudio de habilidades en lugar de competencias matemáticas ya que las consideramos como el pilar fundamental de las mismas y son unidades de análisis más asequibles.

Por otro lado, de manera progresiva, las tecnologías de la información y la comunicación se están incorporando en los centro de enseñanza en todos los niveles educativos, incluida la educación superior; aunque la mera presencia de éstas no aseguran la mejora de los procesos de enseñanza y aprendizaje (Muñoz Carril y González Sanmamed, 2011). De allí nuestro interés en conocer sobre el desarrollo de habilidades que tiene lugar mediante determinadas actividades matemáticas diseñadas para efectuarse con software Mathematica ${ }^{\circledR}$ a realizarse en un taller de informática de la cátedra Análisis Matemático I.

Para poder profundizar en la temática de estudio se analizaron documentos sobre investigaciones relacionadas destacándose el trabajo de Contreras De La Fuente, Font Moll, García Armenteros, Luque Cañada, Marcolini Bernardi, Ordóñez Cañada, Ortega Carpio y Sánchez Gómez (2005) quienes realizaron una investigación sobre la aplicación del programa Mathematica®a las prácticas de Cálculo en el primer año universitario, llegando a la conclusión de que el uso de dicho entorno informático no garantiza resultados satisfactorios en la enseñanza y aprendizaje de los conceptos de límite, continuidad y derivada de una función. En contraposición con estos resultados, Cuicas Ávila, Debel Chourio, Casadei Carniel y Álvarez Vargas (2007) diseñaron lo que denominan "estrategias instruccionales" con software Maple® para favorecer habilidades del pensamiento cognitivas y metacognitivas sobre el concepto de integral definida, logrando un buen rendimiento en los alumnos que participaron de esa experiencia. En Falsetti, Favieri, Scorzo y Williner (2009) se reporta un estudio en el que se diseñaron actividades con uso de software para promover el desarrollo de ciertas habilidades matemáticas en el contexto de un taller con uso de software y el principal resultado fue que, las habilidades promovidas con mayor frecuencia por las actividades del taller, incrementaron sus porcentajes de desarrollo. La información obtenida a través de este estudio fue de utilidad para ajustar las nuevas actividades del taller con un sustento empírico y teórico sólido, para orientarlas a la adquisición y fortalecimiento de habilidades matemáticas.

Por su parte Yániz Álvarez (2006), quien también se refiere a competencias conectándolas con habilidades, señala que para proporcionar una formación que permita adquirir determinadas competencias es necesario diseñar situaciones de aprendizaje que promuevan la adquisición de habilidades, de actitudes y conocimientos que componen esas competencias.

Estos antecedentes nos llevan a la necesidad de indagar sobre el desarrollo de habilidades en el marco de diferentes tipos de tareas diseñadas para hacer uso de software específico y promover habilidades en el ámbito cognitivo. Pensamos que obtener datos sobre esto brindaría un soporte empírico sólido para un diseño de las actividades didácticas más acorde a las habilidades matemáticas que se pretenden promover. De ahí que, el objetivo principal de la investigación que reportamos aquí es conocer sobre el desarrollo de habilidades que tiene lugar mediante tareas que promueven actividad matemática específica y que fueron diseñadas para realizarse con software matemático.

Diseñamos diferentes tipos de actividades con software Mathematica ${ }^{\circledR}$ con el firme propósito de favorecer el desarrollo de habilidades matemáticas en relación con distintos contenidos de Análisis Matemático en una variable. Luego, como segunda etapa, implementamos dichas actividades en el contexto de un taller de la asignatura; y por último efectuamos distintos análisis cuantitativos para identificar habilidades matemáticas desarrolladas en los es- 
tudiantes al momento de resolver los tipos de actividades propuestos.

\subsection{Marco Teórico}

\subsubsection{Habilidades matemáticas}

Una habilidad matemática es la capacidad de efectuar o realizar una tarea matemática eficientemente o de actuar adecuadamente frente a una situación en la que la Matemática esté involucrada, comprendiendo más de una operación intelectual. Las habilidades están ligadas al "saber hacer", y por lo tanto al conocimiento, ya que el "saber hacer" comporta conocer el objeto sobre el que se está actuando así como los mejores modos de actuar sobre él (Hernández Fernández, Delgado Rubí y Fernández De Alaiza, 1998). Al enfocarnos en el desarrollo de habilidades no podemos dejar de establecer conexiones con la Taxonomía de Bloom (Bronk, 2009) que, aunque su versión inicial data de hace más de cincuenta años, aún tiene vigencia y fue reformulada para la "era digital" (Churches, 2009). La clasificación inicial de Bloom se realiza en seis categorías básicas dentro del ámbito cognitivo según la función de la acción en la que la habilidad se manifiesta: conocimiento, comprensión, aplicación, análisis, síntesis y evaluación.

En esta investigación, decidimos utilizar la clasificación de habilidades matemáticas que realizan Hernández et al. (1998), ya que las agrupan no solo de acuerdo al tipo de función que realizan las acciones que manifiestan la habilidad, sino también al objeto matemático con el que se trata al realizar la acción. Consideramos adecuada esta clasificación por ser específica de la actividad matemática, y a su vez porque no establece niveles de jerarquía de acuerdo a la complejidad de la habilidad como lo hace, por ejemplo, la Taxonomía de Bloom. También tuvimos en cuenta varias experiencias educativas que se basan en la clasificación de Hernández y Delgado Rubí (Modarelli, Nolasco, Boucíguez, Irassar, Suárez y Berrino, 2006; Katz y Cámara, 2002; Elena y Herrera, s.f.; Rodríguez, Carnelli y Formica, 2005).

De acuerdo a esta clasificación, tenemos:

Habilidades conceptuales: aquellas que operan directamente con los conceptos (Identificar, Fundamentar, Comparar, Demostrar).

Habilidades traductoras : aquellas que permiten pasar de un dominio a otro del conocimiento o de representación (Interpretar, Modelar, Recodificar).

Habilidades operativas: funcionan generalmente como auxiliares de otras más complejas y están relacionadas con la ejecución en el plano material o verbal (Graficar, Algoritmizar, Aproximar, Optimizar, Calcular).

Habilidades heurísticas: aquellas que emplean recursos heurísticos y que están presentes en un pensamiento reflexivo, estructurado y creativo (Resolver, Analizar, Explorar).

Habilidades metacognitivas: las que son necesarias para la adquisición, empleo y control del conocimiento y demás habilidades cognitivas (Planificar, Predecir, Verificar, Comprobar, Controlar).

Si bien esta clasificación es diferente a la de la Taxonomía de Bloom, podemos efectuar una comparación entre las mismas. Por ejemplo, en el nivel de conocimiento, donde se recuerda la información o conceptos aprendidos, puede considerarse la habilidad Identificar. En el nivel de comprensión, en el que se demuestra el entendimiento de hechos e ideas organizando, comparando, traduciendo, interpretando, haciendo descripciones y exponiendo las ideas principales, pueden considerarse habilidades como las traductoras y Comparar. En el nivel de aplicación, donde se 
hace uso de la información en situaciones nuevas y en la solución de problemas, habilidades como las operativas y Resolver. En los niveles de análisis, síntesis y evaluación, habilidades como Analizar y Explorar.

Además del tipo de habilidad, consideramos la misma estrechamente asociada al contenido, (Falsetti et al., 2009). Esto es así ya que cada habilidad presenta diferentes niveles de dificultad de acuerdo al contexto en el cual se aplica. Por ejemplo, no tiene el mismo nivel de dificultad Analizar el dominio de una función, que Analizar qué discontinuidades posee la misma. Como consecuencia de ello obtuvimos una clasificación del tipo habilidad-contenido que es la utilizada en el presente estudio y que decidimos presentar a medida que realizamos el análisis.

\subsubsection{Uso del software en la enseñanza de la Matemática}

El problema que surge al integrar tecnologías en la enseñanza de la Matemática, en nuestro caso Análisis Matemático, no está en discusión, pero si lo está el cómo llevarlo a la práctica, y qué habilidades fomenta o no la utilización de las mismas (Goldenberg, 2000).

Hitt (2003) asegura que el uso de tecnologías favorece y facilita las diferentes representaciones de los objetos matemáticos, que son necesarias para construir un conocimiento matemático. Uno de los conceptos que remarca como importante es el de visualización matemática. Explica el autor que para comprender el enunciado de un problema matemático se ponen en juego diferentes representaciones de la cuestión a tratar en él. Es importante tener en cuenta las dificultades que manifiestan los alumnos para manipular distintas representaciones y a su vez señala que los profesores no tenemos que priorizar alguna de ellas, en detrimento de otras, en el proceso de aprendizaje de un determinado concepto.

Hitt (2003) hace referencia a otras investigaciones que muestran que los alumnos se resisten a utilizar diferentes representaciones para poder construir un concepto matemático. Este comportamiento se extiende a todos los niveles educativos. Teniendo en cuenta estas investigaciones el autor señala que es importante desarrollar habilidades de visualización en los estudiantes, por ejemplo, haciendo uso de software. Así si un concepto dado en el lenguaje algebraico no es comprendido por el alumno, se puede provocar en el estudiante una mirada diferente de dicho concepto realizando su representación gráfica y quizás, aquello que resultó ser una interpretación errónea, se pueda corregir o rearmar.

Siguiendo esta misma línea Macías Ferrer (2007) remarca que la Matemática se vale de la semiótica y la visualización para representar los entes matemáticos, y que ésta no es un fin en sí mismo sino un medio para mejorar la comprensión de dichos entes abstractos. Este proceso de visualización puede realizarse con lápiz y papel, pero el uso de tecnología lo torna más ágil y efectivo.

La tecnología puede ayudar a los alumnos a aprender Matemática, ya que pueden examinar ejemplos o formas de representar los conceptos de manera más amplia que podrían hacerlo manualmente, facilitando la exploración y realizando algunas conjeturas. El poder gráfico de estas herramientas permite el acceso a modelos visuales poderosos; y el aprendizaje se ve favorecido por la retroalimentación brindada por la tecnología, ya que permite modificar gráficos en la pantalla, observar las consecuencias de un valor dependiente de algún parámetro, transformaciones dinámicas, etc. (NCTM, 2003). La tecnología es una herramienta y su uso adecuado depende del docente, quien debe utilizarla para mejorar las oportunidades de aprendizaje de sus alumnos, seleccionando o creando tareas matemáticas que aprovechen lo que la tecnología ofrece: imágenes visuales de ideas matemáticas, organización y análisis de los datos y cálculos en forma eficiente, entre otros. (NCTM, 2003).

En particular el software Mathematica® permite realizar computación simbólica y numérica al mismo tiempo, esto es una característica importante para poder resolver problemas vinculados con aplicaciones en el campo de la ingeniería asegurando resultados consistentes que permiten dar respuesta a dichas problemáticas. Además este software 
permite programar en diferentes estilos como ser: basado en líneas, procedimental, recursiva, funcional, entre otros.

\subsubsection{Clasificación de actividades}

Seguimos la tipología brindada por Delgado Rubí en el curso de "Resolución de Problemas, aprendizaje matemático y uso de TICs" que dictó en la Universidad Nacional de La Matanza en diciembre de 2006. Este autor clasifica las actividades que se pueden llevar a cabo en el aula de Matemática en:

Actividades de generalización: son aquellas en las que el alumno debe explorar para poder extraer un resultado, conclusión o propiedad referente al estudio de casos particulares, que luego extenderá (generalizará) a un determinado universo.

Actividades de estudio de casos: en las cuales se trabaja con parámetros o con otro objeto matemático que pueda presentar diferentes situaciones.

Actividades de aplicación de resultados teóricos a problemas prácticos: son las que se resuelven reflejando la teoría estudiada.

Actividades de aplicación de algoritmos dados y conocidos: en las que se realizan cálculos, pasos algebraicos, resoluciones varias aplicando algoritmos conocidos.

Actividades de construcción: en las que el alumno debe brindar un ejemplo que él mismo haya inventado, armado o construido.

\subsection{Contexto de la Investigación}

Las actividades se realizaron en el taller con uso del software Mathematica®integrado a las asignaturas Cálculo I y Análisis Matemático I del primer año de las carreras de Ingeniería Industrial, Electrónica e Informática de la Universidad Nacional de La Matanza, provincia de Buenos Aires. Elegimos Mathematica® por las razones que explicitamos a continuación. La Universidad cuenta con licencia del programa y además consideramos que es un potente software, que permite hacer cálculos de Matemática avanzada en forma rápida y gráficos precisos, favoreciendo el análisis, la inferencia y la justificación de los temas estudiados. Este software fue concebido con fines técnico-científicos y pretendemos que el alumno utilice la tecnología como herramienta cognitiva, es decir, como medio facilitador del aprendizaje, entonces una de nuestras funciones es la de diseñar e implementar una situación, que involucre actividades para resolver y estudiar, movilizando el conocimiento enseñado en las clases de la asignatura.

Desde el punto de vista del alumno, la capacitación en el uso del software se realiza en el ámbito del mencionado taller, el cual complementa las clases teórico-prácticas, que se dictan en forma tradicional, expositivo-dialogada, con la realización de ejercicios modélicos orientados por una guía impresa común a todas las comisiones. Las actividades de los talleres son obligatorias para todos los alumnos y se organizan en trabajos prácticos que deben resolverse usando el software mencionado. Los alumnos reciben una capacitación sobre el software en diferentes modalidades: presencial y tutorial con instructivos especialmente diseñados, y de acuerdo a la elección del alumno pueden optar por una u otra forma. Tanto el material didáctico como las actividades de taller y la atención y orientación a los alumnos son llevados a cabo por integrantes del equipo que realizó la presente investigación. 
Dentro de la bibliografía recomendada en la asignatura contamos con los libros de Stewart (1999), Larson, Hostetler y Edwars (2002), Piskunov (1977), entre otros. El régimen de cursado de la materia es anual con dos exámenes parciales. La acreditación se logra por promoción (aprobación de los dos parciales con nota mayor o igual que siete) o por examen final (aprobación de los dos parciales con nota entre 4 y 6 puntos). En ambos casos (promoción o examen final) el alumno debe aprobar los trabajos prácticos efectuados con software Mathematica ${ }^{\circledR}$. Esto significa que debe aprobar tanto los que son presentados en forma impresa (que efectúan en forma grupal), como así también una evaluación oral e individual (realizada a fin de año) que consiste en un interrogatorio estructurado sobre temas de la asignatura ejecutados con el software.

\subsection{Metodología utilizada para el estudio propuesto.}

Este es un estudio transeccional debido a que la recolección de datos se realiza en un solo momento, en nuestro caso en la corrección de los trabajos prácticos de los alumnos. Dentro del tipo transeccional este diseño es descriptivointerpretativo ya que tiene por objetivo tener un acercamiento a la manifestación de habilidades matemáticas de acuerdo a la actividad matemática realizada y describirlas (Gómez, 2006). La muestra seleccionada es no probabilística, pues las producciones de los alumnos provienen de los cursos en las cuales las docentes de este grupo de investigación dictan sus clases, resultando un total de 9 comisiones y 133 producciones escritas.

Dividimos la investigación en tres etapas

\subsubsection{Diseño de actividades siguiendo la tipología establecida en el marco teórico y poniendo énfasis en el desarrollo de habilidades}

Las actividades a resolver con el software Mathematica ${ }^{\circledR}$ las diseñamos especialmente de acuerdo a la clasificación brindada en el marco teórico y poniendo especial atención en el desarrollo de habilidades matemáticas. Las mismas fueron confeccionadas por el grupo de docentes que participan de este estudio y se han dividido en tres trabajos prácticos a resolver por el alumno durante el ciclo lectivo. Para aprender el uso del software y realizar estas actividades, los alumnos cuentan con el taller de asistencia no obligatoria que mencionamos anteriormente.

En relación a la clasificación de actividades presentadas en el marco teórico propusimos los siguientes ejercicios:

- Actividad de generalización: ejercicio sobre contracciones y dilataciones horizontales de funciones.

- Actividad de estudio de casos: ejercicio sobre función racional: máximo dominio real y ecuaciones de las asíntotas verticales.

- Actividad de aplicación de resultados teóricos a problemas prácticos: ejercicio sobre transformaciones geométricas de función homográfica.

- Actividad de aplicación de algoritmos dados y conocidos: ejercicio sobre funciones con raíces cuadradas en el denominador: máximo dominio real, raíces, asíntotas y continuidad de una función.

- Actividad de construcción: ejercicio sobre límite, continuidad y asíntotas.

\subsubsection{Análisis preliminar de las habilidades promovidas por los ejercicios del primer trabajo práctico}

Dado que las actividades fueron diseñadas pensando en promover ciertas habilidades, antes de administrarlas a los estudiantes realizamos un análisis preliminar de las mismas. Este análisis consistió en determinar, en la resolución 
de cada actividad, qué tipo de habilidad está presente, para asegurarnos que efectivamente podrían surgir las habilidades propuestas y para saber además cuáles otras podrían manifestarse. Cabe aclarar que el mencionado análisis fue realizado por el grupo de docentes que intervino en la investigación en forma conjunta, es decir, discutiendo y examinando las habilidades matemáticas en cada paso de la resolución de cada actividad.

\subsubsection{Análisis de los trabajos prácticos realizados por los alumnos de nueve comisiones}

Examinamos las producciones de los alumnos y recolectamos dicha información en planillas diseñadas especialmente para este estudio. Las planillas son matrices cuyas filas representan a cada uno de los alumnos cuyos trabajos fueron estudiados y las columnas cada una de las habilidades analizadas que responden al análisis preliminar. Para la evaluación de cada habilidad establecimos las categorías: Mal o No responde, Regular, Bien, de acuerdo a las respuestas dadas para cada ejercicio solicitado en los trabajos.

Para realizar el análisis cuantitativo asignamos códigos numéricos a cada categoría de la evaluación. Determinamos con 1 a la categoría Mal o No Responde, con 2 a la Regular y con 3 a la categoría Bien, de esta manera pudimos hacer un análisis descriptivo que nos permita puntualizar en detalle las características presentes en cuanto a las habilidades matemáticas y la actividad matemática específica realizada.

\subsection{Sistematización y Análisis de datos}

Efectuamos el análisis de 133 producciones escritas. Presentamos una actividad de cada tipo en la clasificación y evaluamos las habilidades promovidas por la misma.

Decidimos realizar dos tipos de análisis estadísticos sobre los datos recolectados de las producciones de los alumnos. En primer lugar un análisis descriptivo para poder puntualizar la manera en que las distintas habilidades matemáticas se manifiestan en cada tipo de actividad matemática diseñada para resolver utilizando el software.

En segundo lugar, dado que los datos son multivariados, consideramos apropiado realizar un análisis factorial por actividad ya que esta técnica estadística permite determinar grupos homogéneos de variables, que suelen denominarse factores. La misma puede ser usada tanto en forma exploratoria, para tratar de descubrir la estructura interna de un conjunto de variables; o de forma confirmatoria, con el objetivo de determinar si los factores obtenidos se corresponden con los que cabría esperar a la luz de una teoría previa acerca de los datos.

Debido a que en nuestra investigación anterior habíamos podido establecer una fuerte relación entre las habilidades matemáticas y los contenidos matemáticos; más la intuición al corregir las producciones de los alumnos que esta relación seguía vigente, es que consideramos en utilizar esta técnica en su forma confirmatoria con el fin de las hipótesis establecidas a priori.

La medida de adecuación de la muestra, el índice de Kaiser-Meyer-Olkin (KMO) permite determinar la idoneidad de los datos para realizar un análisis factorial. Dicho índice varía entre 0 y 1 y los valores más cercanos a 1 son los que garantizan mejores resultados al realizar el análisis factorial. Al aplicar el índice a nuestra muestra, los valores obtenidos estaban en el rango comprendido entre 0,5 y 0,75; esto nos permite, de manera aceptable, realizar dicho análisis en forma confirmatoria como dijimos anteriormente. (Hernández Rodríguez, R., 1998) 


\subsubsection{Actividad de generalización}

\section{Tema: Contracciones y dilataciones horizontales de funciones}

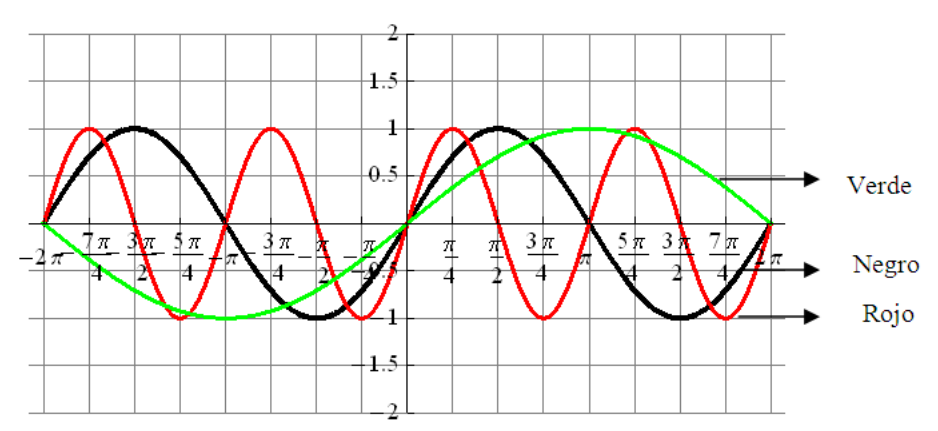

Figura 1.1

En la gráfica dada en la figura 1, la curva que está dibujada en color negro corresponde a $y=\operatorname{sen} x$ graficada en el intervalo $[-2 \pi, 2 \pi]$.

-Auxiliándote con Mathematica decide cómo se puede modificar la ecuación y $=\operatorname{sen} x$ para que el gráfico de dicha ecuación corresponda a la curva que se presenta en rojo. ¿Y para que sea la que está en verde?

-¿Qué sucede si dicha operación matemática es aplicada a cualquier otra función?

Consideramos a esta una actividad de generalización ya que, a partir del estudio de dilataciones y contracciones horizontales en la función seno, se pretende que el alumno pueda inferir el comportamiento de este tipo de transformaciones en otras funciones, para luego lograr una generalización. Esta actividad fue diseñada teniendo en cuenta la bibliografía usual para cursos de Precálculo en la cual las funciones trigonométricas, por sus características de periodicidad, imagen acotada, etc., son consideradas como prototipos para mostrar las transformaciones estudiadas.

\section{Análisis descriptivo}

Las habilidades promovidas por esta actividad, de acuerdo al análisis preliminar efectuado, son: Recodificar - Explorar - Comparar - Generalizar

Vinculamos cada habilidad en relación con el contenido, obteniendo:

- Recodificar contracciones y dilataciones horizontales en gráficos de función seno.

- Explorar contracciones y dilataciones horizontales en gráficos de función seno.

- Comparar contracciones y dilataciones horizontales en gráficos de función seno.

- Explorar contracciones y dilataciones horizontales en gráficos de una función cualquiera.

- Generalizar contracciones y dilataciones horizontales en gráficos de una función cualquiera.

En esta actividad, evaluamos con calificación "Bien" (B) al alumno que: 
- Recodifica al lenguaje analítico lo observado en el gráfico.

- Explora y determina los coeficientes correctos para obtener las curvas en color rojo y verde.

- Compara las tres funciones y establece el efecto que produce multiplicar por una constante el argumento de la función seno. Se establece de acuerdo al valor del parámetro, la contracción o la dilatación de la gráfica. También se calificó (B) cuando se hizo la relación correcta entre parámetro y variación del período.

- Explora y muestra gráficos de otras funciones en las cuales realiza la misma transformación.

- Generaliza en forma correcta lo que le sucede a una función cualquiera $f(x)$ al multiplicar su argumento por una constante, considerando todos los casos posibles para el valor de dicha constante.

En el caso que alguno de estos aspectos no esté completamente bien, evaluamos con "Regular" y si no responde o lo efectuado no es correcto, consideramos como calificación "Mal".

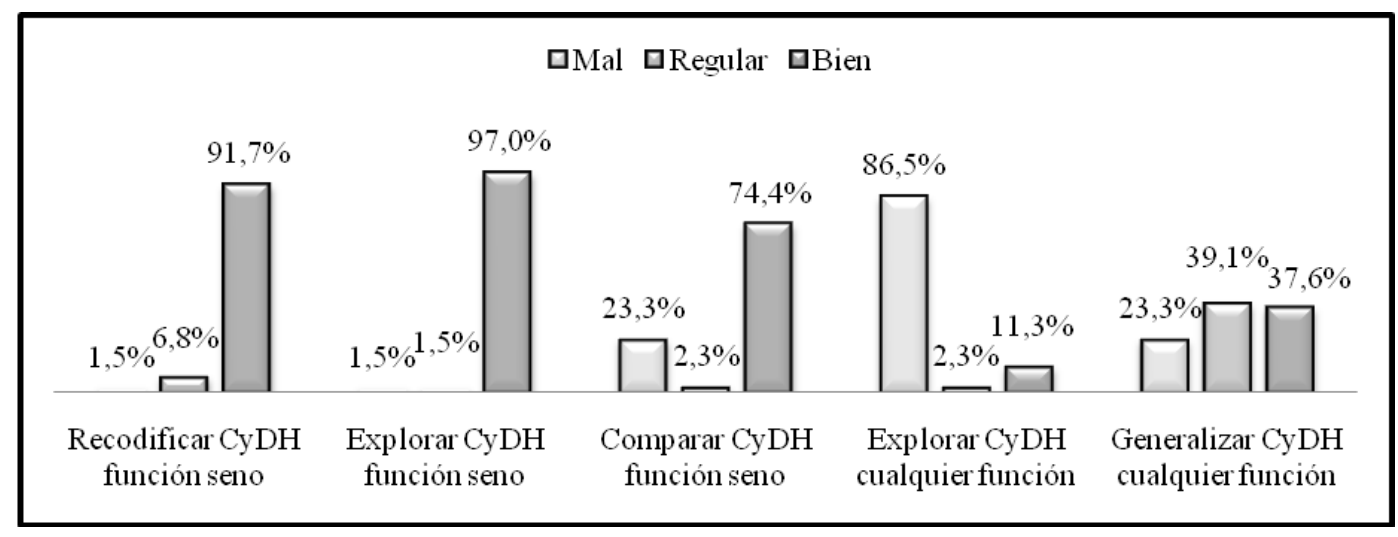

Figura 1.2 Gráfico de distribución de porcentajes de desempeño de cada habilidad-contenido.

Podemos observar desde el gráfico, altos porcentajes de buen desempeño en las habilidades Recodificar, Explorar y Comparar contracciones y dilataciones horizontales en gráficos de función seno (la abreviatura usada en el gráfico es Recodificar C y DH función seno, Explorar C y DH función seno, Comparar C y DH función seno, respectivamente).

La habilidad Explorar contracciones y dilataciones horizontales en gráficos de una función cualquiera (abreviada en la Figura 2 como Explorar C y DH cualquier función) tiene alto porcentaje en la categoría mal o no responde. A pesar de la potencialidad del software para realizar exploraciones que ayudaran al alumno a responder la consigna, esta habilidad tuvo bajo desempeño.

Con respecto a Generalizar contracciones y dilataciones horizontales en gráficos de una función cualquiera (abreviada en la Figura 2 como Generalizar C y DH cualquier función), los resultados de las categorías Bien y Regular presentan valores similares. En esta oportunidad, en la generalización, observamos la deficiencia en la expresión en lenguaje escrito para poder formular el comportamiento de lo analizado y la no consideración de todos los casos posibles.

Los gráficos muestran una diferencia considerable respecto al buen desempeño en las habilidades consideradas entre el caso particular de las funciones trigonométricas, donde han respondido bien a las consignas, y el caso en el que se solicita la generalización para cualquier función. Esta actividad fue diseñada teniendo en cuenta la bibliografía usual para cursos de Precálculo en los cuales las funciones trigonométricas, por sus características de periodicidad, imagen acotada, etc., son consideradas como prototipos para mostrar las transformaciones estudiadas. Los resultados nos muestran que el trabajo con un ejemplo prototípico no siempre es suficiente para que el alumno pueda generalizar a otros casos. Nuevamente aquí podemos decir que, como señalamos en el marco teórico, la 
construcción de un concepto matemático requiere de diversas representaciones por parte del alumno y que algunas se ven favorecidas por la incorporación de tecnologías pero esto no implica la comprensión del objeto matemático a estudiar.

\section{Análisis factorial de las variables}

En este caso, las dimensiones o variables son las cinco habilidades consideradas y la cantidad de datos por cada dimensión es 133, que toman valores entre 1,2 y 3 según su calificación sea Mal, Regular o Bien. Se analiza entonces una matriz de $5 \times 133$ datos. El análisis factorial realizado y procesado con SPSS®arrojó, con un $69 \%$ de la varianza explicada, en dos componentes.

\begin{tabular}{|l|l|l|}
\hline & \multicolumn{2}{l|}{ Componentes } \\
\cline { 2 - 3 } & 1 & 2 \\
\hline Recodificar contracciones y dilataciones horizontales en gráficos de función seno. & 0,925 & 0,175 \\
\hline Explorar contracciones y dilataciones horizontales en gráficos de función seno & 0,860 & 0,261 \\
\hline Comparar contracciones y dilataciones horizontales en gráficos de función seno & 0,588 & $-0,542$ \\
\hline $\begin{array}{l}\text { Explorar contracciones y dilataciones horizontales en gráficos de una función } \\
\text { cualquiera }\end{array}$ & 0,254 & $-0,594$ \\
\hline $\begin{array}{l}\text { Generalizar contracciones y dilataciones horizontales en gráficos de una función } \\
\text { cualquiera }\end{array}$ & 0,098 & 0,838 \\
\hline
\end{tabular}

Tabla 1.1

Los valores de cada componente se pueden interpretar como los "pesos" que cada habilidad tiene en esa componente y con ellos se conforma la nueva variable. Es decir, el fenómeno podría reducirse a considerar dos variables compuestas del siguiente modo:

$$
\begin{aligned}
& y_{1}=0,925 x_{1}+0,860 x_{2}+0,588 x_{3}+0,254 x_{4}+0,098 x_{5}, \\
& y_{2}=0,175 x_{1}+0,261 x_{2}-0,542 x_{3}-0,594 x_{4}+0,838 x_{5}
\end{aligned}
$$

Donde $x_{1}, x_{2}, x_{3}, x_{4}, x_{5}$ son los valores resultantes de la evaluación de las habilidades consideradas.

En cada componente tienen mayores pesos las habilidades referidas a uno de los dos temas tratados en este ejercicio. En la primera componente, los mayores pesos los tienen las variables o dimensiones Recodificar, Explorar y Comparar contracciones y dilataciones horizontales en gráficos de función seno. La segunda componente tiene por elementos principales a la habilidad Generalizar contracciones y dilataciones horizontales en gráficos de una función cualquiera. Interpretamos los valores negativos en las habilidades Comparar y en Explorar contracciones y dilataciones horizontales en gráficos de una función cualquiera, que tienen alto valor absoluto, como que los alumnos no dejan suficiente evidencia, en sus trabajos de la exploración en otras funciones como paso previo a la generalización, lo que llama la atención dado que sí dejan evidencia de la exploración cuando trabajan con la función seno y además lo hacen bien, según lo visto en el análisis descriptivo anterior.

Vemos en este caso que, en cuanto a las habilidades, hay dos agrupamientos: por un lado las que se refieren a la función seno en particular, observemos en la Figura 2 que son además las que tienen porcentajes más altos en la categoría Bien, y por el otro, las vinculadas con la generalización para las cuales los alumnos obtuvieron menor calificación. La primera componente nos revela que los pesos correspondientes a las habilidades asociadas a una función cualquiera (las dos últimas) son bastante menores de los pesos correspondientes a las habilidades asociadas a la función seno (las dos primeras). Esta observación refuerza nuestra hipótesis extraída en el análisis descriptivo 
precedente respecto a que no siempre es eficiente el uso de funciones prototipo para la generalización, aún cuando es un recurso muy explotado en los libros de texto usuales de Cálculo como el de Stewart (1999), o el de Larson et al. (2002).

\subsubsection{Actividad de estudio de casos}

Esta actividad trata de romper la conceptualización errada que en las funciones racionales existen asíntotas verticales en todas las raíces del denominador.

\section{Tema: Función Racional: dominio y asíntotas verticales.}

Para cualquier función racional cuya fórmula es del tipo $f(x)=\frac{P_{1}(x)}{P_{2}(x)}$ donde $P_{1}(x)$ y $P_{2}(x)$ son polinomios, se calcula su dominio planteando $P_{2}(x)=0$. Las soluciones de esta ecuación son valores de abscisa donde la función tiene asíntotas verticales.

¿Por qué se afirma que el dominio se calcula planteando $P_{2}(x)=0$ ?

Decidir si con las condiciones dadas es válido concluir la afirmación subrayada.

¿Te auxiliaste con el Mathematica®para decidir? ¿Cómo?

Consideramos a esta actividad como un estudio de casos, debido a que son dos las posibilidades que se presentan en los ceros del denominador de una función racional, respecto a la existencia o no de asíntota vertical. Por un lado puede ser que en la raíz calculada exista una discontinuidad evitable, por el otro puede suceder que la función presente una asíntota vertical en dicho punto. De los dos casos, el primero es el que brinda el contraejemplo para justificar que la proposición subrayada es falsa.

\section{Análisis descriptivo}

Las habilidades promovidas por la actividad, en este caso, son: Identificar - Fundamentar - Explorar - Identificar

La vinculación de cada habilidad en relación con el contenido que efectuamos en este caso es:

- Identificar máximo dominio real de una función racional expresada de la forma $f(x)=\frac{P_{1}(x)}{P_{2}(x)}$

- Fundamentar la definición de máximo dominio real debido a que no está definida la división por cero.

- Explorar asíntotas verticales en funciones racionales.

- Identificar asíntota vertical.

- Fundamentar la existencia o no de asíntota vertical.

En esta actividad, consideramos una calificación "Bien" (B) al alumno que:

- Determina correctamente el máximo dominio real de la función. 
- Argumenta que excluye del conjunto de números reales a las raíces del denominador por no existir la división por cero.

- Ejemplifica con diferentes tipos de funciones para poder determinar la veracidad o falsedad de la proposición dada.

- Brinda un contraejemplo adecuado para argumentar que la premisa dada es falsa (en cuyo caso identifica el concepto de asíntota vertical).

En el caso que alguno de estos aspectos no estén completamente bien, evaluamos con "Regular" y si no responde o lo efectuado no es correcto, consideramos como calificación "Mal".

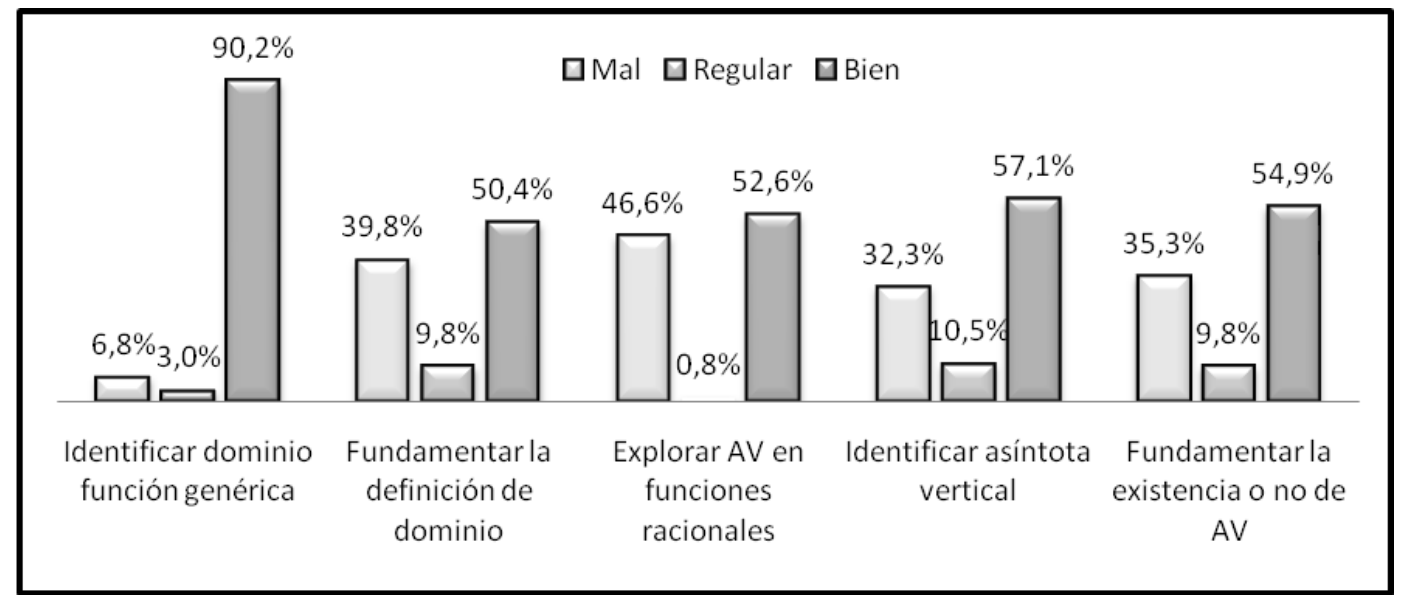

Figura 1.3 Gráfico de distribución porcentajes de desempeño de cada habilidad-contenido

La mayoría de los alumnos identifican el dominio de una función racional dada en forma genérica como cociente de polinomios. Las demás habilidades no poseen niveles altos de desarrollo como ésta. En el caso de fundamentar la existencia o no de asíntota vertical en puntos que son raíces del denominador, muchos alumnos incurren en el error por considerar como propiedad verdadera que si un valor anula el denominador entonces allí hay una asíntota vertical, es decir no recurren a la definición de asíntota vertical con el límite infinito en al menos uno de los laterales. De aquí también los porcentajes altos entre Mal-Regular en las habilidades asociadas con este conocimiento. Si bien tuvieron la posibilidad de explorar con el software (NCTM, 2003), no pudieron encontrar ejemplos variados que den los distintos casos que se pueden presentar. Al respecto, consideramos que desde el diseño de la tarea, sería necesario incluir mayor orientación que guíe al alumno en la exploración.

\section{Análisis factorial de las variables}

A través del análisis factorial realizado pudimos lograr un agrupamiento en dos componentes con un $69 \%$ de la varianza explicada.

Esto significa que en lugar de trabajar con las cinco variables dadas podríamos reducirlas a dos variables compuestas del siguiente modo:

$$
\begin{gathered}
y_{1}=-0,026 x_{1}+0,378 x_{2}+0,392 x_{3}+0,961 x_{4}+0,969 x_{5} \\
y_{2}=0,818 x_{1}+0,715 x_{2}-0,335 x_{3}-0,073 x_{4}-0,049 x_{5}
\end{gathered}
$$




\begin{tabular}{|l|l|l|}
\hline & \multicolumn{2}{l|}{ Componente } \\
\cline { 2 - 3 } & 1 & 2 \\
\hline Identificar dominio de una función expresada genéricamente & $-0,026$ & 0,818 \\
\hline $\begin{array}{l}\text { Fundamentar la definición de dominio debido a la no existencia de la división por } \\
\text { cero. }\end{array}$ & 0,378 & 0,715 \\
\hline Explorar asíntotas verticales en funciones racionales & 0,392 & $-0,335$ \\
\hline Identificar asíntota vertical & 0,961 & $-0,073$ \\
\hline Fundamentar la existencia o no de asíntota vertical & 0,969 & $-0,049$ \\
\hline
\end{tabular}

Tabla 1.2

La primera componente agrupa las habilidades relacionadas con las asíntotas verticales de la función racional: identificar asíntota vertical y fundamentar la existencia o no de asíntota vertical. La segunda componente está relacionada con las habilidades vinculadas con el dominio de una función racional: identificar máximo dominio real de una función expresada de la forma $f(x)=\frac{P_{1}(x)}{P_{2}(x)}$ y fundamentar la definición de dominio debido a la no existencia de la división por cero.

Observamos que la agrupación se hizo de acuerdo al tema de función racional con el que están vinculadas, es decir, por un lado las referidas a dominio de una función racional y, por otro lado, las que tratan sobre asíntotas verticales de dichas funciones.

Además, afortunadamente Fundamentar acompaña a Identificar en cada caso, esto es destacable si se tiene en cuenta que el enunciado de la actividad es sumamente genérico y exige relacionar el planteo de una ecuación con definiciones de dominio y asíntota respectivamente. Consideramos que las habilidades identificadas a priori en el análisis preliminar fueron evidenciadas con buen grado de desempeño al realizarse esta actividad, lo que nos sugiere que es una actividad útil para el desarrollo de las mismas.

\subsubsection{Actividades de aplicación de resultados teóricos a problemas prácticos}

\section{Tema: Transformaciones geométricas de función homográfica}

¿Qué operaciones matemáticas son necesarias para que el gráfico de $y=1 / x$ se desplace 5 unidades hacia la derecha, 2 unidades hacia abajo y sufra una contracción vertical de 3 unidades?

Graficar la función $y=1 / x$ y la transformada.

Clasificamos esta actividad dentro de la tipología "Aplicación de resultados teóricos a problemas prácticos" debido a que en las clases teóricas de la asignatura se explican las diferentes transformaciones que se pueden realizar al gráfico de una función (traslaciones, dilataciones, reflexiones, etc.). Estos resultados son los que el alumno deberá tener en cuenta para brindar la expresión analítica de la función solicitada y controlar gráficamente lo obtenido.

\section{Análisis descriptivo}

En este caso, las habilidades promovidas por la actividad son: Identificar - Controlar

La vinculación efectuada en cada habilidad en relación con el contenido es la siguiente: 
- Identificar operaciones sobre la expresión algebraica en el criterio de una función homográfica que se visualicen como movimientos de su gráfica.

- Controlar que las operaciones sobre la expresión algebraica en el criterio de una función homográfica se visualicen como movimientos de su gráfica.

Consideramos una calificación "Bien" (B) al alumno que:

- Proporciona la expresión analítica correcta de la función transformada con los movimientos dados en la consigna.

- Grafica ambas funciones o la obtenida, para controlar que los movimientos efectuados sean los correctos.

En el caso que alguno de estos aspectos no esté completamente bien, evaluamos con "Regular" (R) y si no responde o lo efectuado no es correcto, consideramos como calificación "Mal" (M)

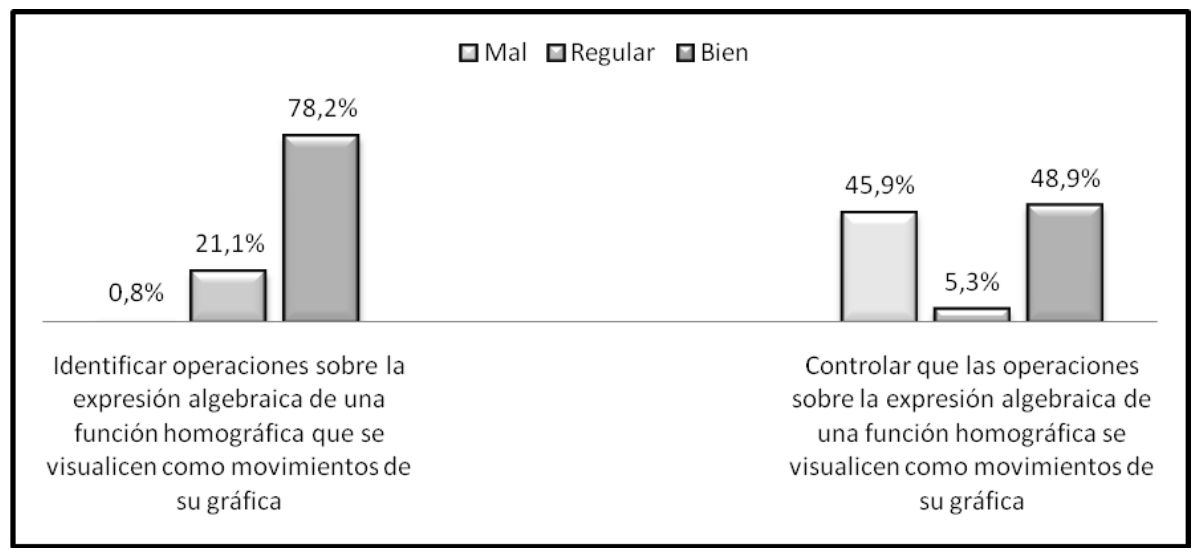

Figura 1.4 Gráfico de distribución de porcentajes de desempeño de cada habilidad-contenido

En general los alumnos identifican las traslaciones, contracciones o dilataciones aplicadas a una función. Con respecto a la habilidad Controlar pretendíamos que, mediante el gráfico que debían realizar, controlaran que habían logrado la función solicitada. Fueron parejos los niveles entre los estudiantes que lo realizaron "Bien" y aquellos que lo hicieron "Mal".

\section{Análisis factorial de las variables}

EL análisis factorial efectuado arrojó un agrupamiento en una sola componente con un 75,6\% de la varianza explicada.

\begin{tabular}{|l|l|}
\hline & Componente \\
\cline { 2 - 2 } & 1 \\
\hline $\begin{array}{l}\text { Identificar operaciones sobre la expresión algebraica de una función homográfica que } \\
\text { se visualicen como movimientos de su gráfica }\end{array}$ & 0,870 \\
\hline $\begin{array}{l}\text { Controlar que las operaciones sobre la expresión algebraica de una función homográ- } \\
\text { fica se visualicen como movimientos de su gráfica. }\end{array}$ & 0,870 \\
\hline
\end{tabular}

Tabla 1.3

Se puede definir entonces una nueva variable, 


$$
y_{1}=0,870 x_{1}+0,870 x_{2}
$$

donde $x_{1}, x_{2}$ son las mediciones de las dos habilidades consideradas.

En el análisis factorial comprobamos que las dos tienen comportamientos similares, es decir, el alumno que realizó bien la transformación solicitada también lo controló gráficamente.

\subsubsection{Actividades de aplicación de algoritmos dados y conocidos}

\section{Tema: Funciones con raíces cuadradas en el denominador: dominio, raíces, asíntotas y continuidad de una función.}

Dada la siguiente función $g$ tal que $g(x)=\frac{x^{2}-9}{\sqrt{x^{2}-9}}$. Se pide:

a) Calcular dominio y ceros.

b) Hallar las ecuaciones de las asíntotas.

c) Estudiar continuidad en [3,12], clasificar si es que existen las discontinuidades

d) Graficar todo en un mismo eje (función y asíntotas), usar diferentes colores. Realizar observaciones si fueran necesarias con referencia al gráfico obtenido.

Consideramos esta actividad como aplicación de algoritmos dados y conocidos. Si bien las cuentas y el cálculo de los límites los efectúa el software, el alumno debe saber aplicar las definiciones y los algoritmos explicados en las clases teórico-prácticas de la asignatura para la resolución de límites, cálculo de asíntotas, cero de la función, y análisis de continuidad en un intervalo cerrado.

\section{Análisis descriptivo}

Las habilidades promovidas por esta actividad son: Identificar - Analizar - Controlar - Calcular y la vinculación de cada habilidad en relación con el contenido, la siguiente:

- Identificar dominio de función.

- Analizar dominio de función.

- Controlar las raíces de la función.

- Identificar asíntotas al gráfico de una función.

- Analizar función para determinar asíntotas.

- Calcular asíntotas.

- Identificar continuidad intervalo cerrado.

En este caso, consideramos una calificación Bien (B) al alumno que: 
- Calculó correctamente el dominio de la función, sus asíntotas, las discontinuidades e identificó continuidad en un intervalo cerrado (en este caso estudiando lateralmente los extremos del intervalo y luego los puntos interiores al mismo).

- Con respecto a las raíces de la función, el software arroja como respuesta a números que no están en el dominio de la misma (son discontinuidades evitables). El alumno debía controlar esta respuesta y, a pesar del resultado del programa, escribir que la función no tiene ceros.

En el caso que alguno de estos aspectos no esté completamente bien, evaluamos con Regular y si no responde o lo efectuado no es correcto, consideramos como calificación Mal.

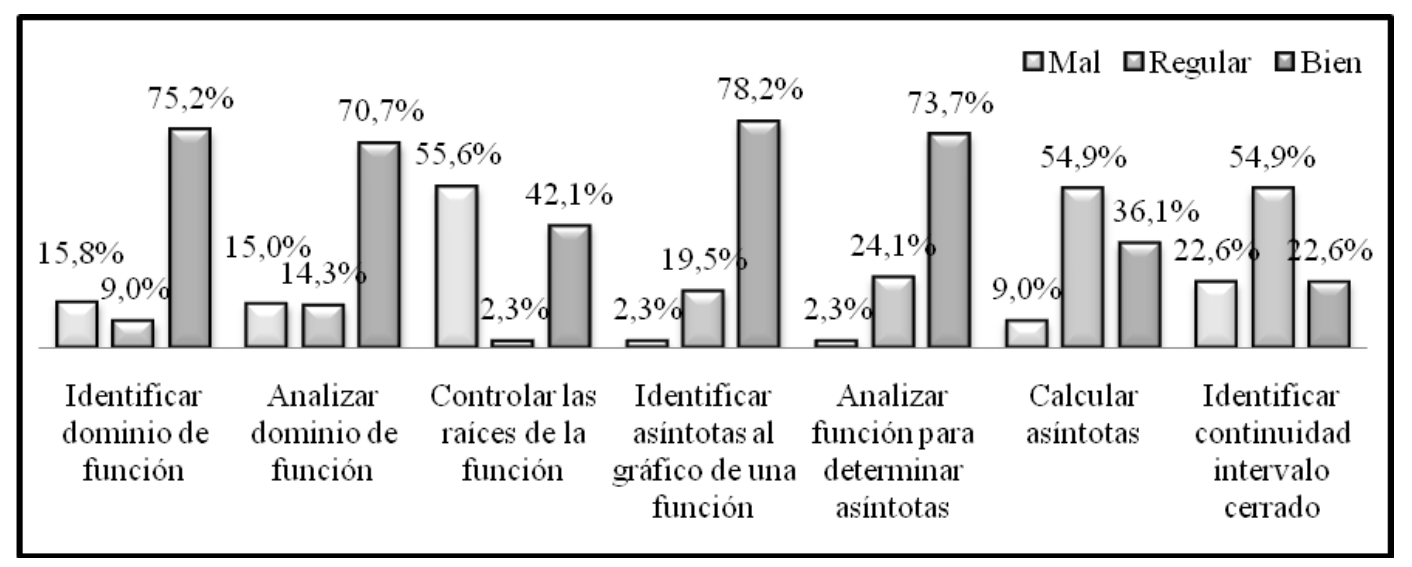

Figura 1.5 Gráfico de distribución de porcentajes de desempeño de cada habilidad-contenido

Los alumnos no tuvieron dificultades en Identificar y Analizar el dominio de la función y sus asíntotas. En general no controlaron que las raíces brindadas por el programa no estaban en el dominio de la función. En el cálculo de las asíntotas hubo dificultad en dos aspectos: por un lado hallar asíntotas verticales cuando en realidad no existían, y por otro calcular asíntota oblicua solo para valores positivos de la variable independiente. En cuanto a Identificar y Analizar continuidad en un intervalo cerrado, muchos olvidaban analizar los extremos de dicho intervalo en forma correcta. En algunos casos no reconocieron lo que se les pedía, interpretando que debían estudiar lo que pasaba solo en esos puntos y no asociándolo al concepto de continuidad en un intervalo cerrado. Los puntos de discontinuidad evitable también fueron conflictivos a la hora de clasificarlos, y a pesar de realizar el gráfico de la función, muchos no tuvieron buen desempeño en la habilidad de Controlar las respuestas dadas.

\section{Análisis factorial de las variables}

Realizamos el análisis factorial para determinar si las variables podrían agruparse de alguna manera, de tal forma que un número menor de las mismas pudiera ser usado para explicar el fenómeno. Este análisis factorial se realizó mediante el método de rotación normalización Varimax con Kaiser, y la rotación ha convergido en 3 iteraciones arrojando el siguiente agrupamiento de dos componentes con un $66,7 \%$ de la varianza explicada.

Nuevamente aquí puede apreciarse cómo se distribuye el peso de las variables de acuerdo al contenido pudiendo reducir el análisis en dos variables, cada una con mayores pesos en cada uno de los dos contenidos tratados:

$$
\begin{aligned}
& y_{1}=0,938 x_{1}+0,955 x_{2}+0,095 x_{3}+0,556 x_{4}+0,506 x_{5}-0,067 x_{6}+0,474 x_{7} \\
& y_{2}=0,096 x_{1}+0,031 x_{2}+0,538 x_{3}+0,697 x_{4}+0,738 x_{5}+0,823 x_{6}+0,258 x_{7}
\end{aligned}
$$




\begin{tabular}{|l|l|l|}
\hline & Componente \\
\cline { 2 - 3 } & 1 & 2 \\
\hline Identificar dominio de función & 0,938 & 0,096 \\
\hline Analizar dominio de función & 0,955 & 0,031 \\
\hline Controlar las raíces de la función & 0,095 & 0,538 \\
\hline Identificar asíntotas al gráfico de una función & 0,556 & 0,697 \\
\hline Analizar función para determinar asíntota & 0,506 & 0,738 \\
\hline Identificar continuidad en intervalo cerrado & $-0,067$ & 0,823 \\
\hline Calcular asíntota oblicua & 0,474 & 0,258 \\
\hline
\end{tabular}

Tabla 1.4

En una componente tienen mayor peso las habilidades Identificar dominio de función con raíz cuadrada en el denominador y Analizar dominio de dicho tipo de función. En la otra componente tienen mayor peso las habilidades Identificar asíntotas al gráfico de una función con raíz cuadrada en el denominador, Analizar la función para determinar asintotas y Calcular asintotas; es decir, las relacionadas con la determinación de las asíntotas de estas funciones.

Consideramos que estos resultados están reafirmando la propuesta de vinculación de la habilidad con el contenido. Es destacable observar que no es lo mismo Identificar y Analizar dominio de funciones polinómicas, que de funciones irracionales o de funciones logarítmicas. Como así también no presenta el mismo nivel de dificultad el determinar asíntotas en funciones que tienen en su expresión analítica una raíz o logarítmicas.

Asimismo esta separación entre las habilidades relativas al dominio de la función y las relativas a las asíntotas, está en relación con el desempeño de los alumnos en la resolución de los ejercicios, ya que el mismo era bueno en el análisis y cálculo del dominio pero regular en el análisis, y cálculo de las asíntotas. Esto nos estaría diciendo que a pesar de que los alumnos son capaces de analizar e identificar el dominio de una función con raíz cuadrada en el denominador, no pueden analizar y calcular las asíntotas de la misma manera. Esto podría deberse a que los alumnos no logran incorporar plenamente la definición de asíntota, ya que suelen concluir que en los puntos que están fuera del dominio seguro existirá asíntota vertical sin usar la definición de asíntota vertical de una función, ya que no están verificando la existencia de la misma a través del límite.

Otra dificultad de este ejercicio reside en que la función posee dos asíntotas oblicuas. En el cálculo de las mismas hemos observado que los alumnos solo calculaban el límite para $x$ tendiendo a $+\infty$. Como consecuencia de ello, obtenían una sola de las asíntotas oblicuas, y al hacer el gráfico descubrían que había otra. Sin embargo, a pesar de visualizar gráficamente las dos asíntotas oblicuas, no planteaban el límite correspondiente para la obtención de esta última.

El dominio de esta función es una unión de intervalos disjuntos; lo que conlleva a que en el cálculo de las posibles asíntotas verticales se realicen los límites laterales correspondientes. Los alumnos distinguían la posibilidad de la misma en aquellos puntos extremos del dominio, pero sin considerar los límites laterales.

Estos valores no pertenecientes del dominio eran considerados a su vez raíces de la función ya que, cuando al programa se le plantea el comando para calcularlas; éste devuelve dichos valores como soluciones. La respuesta generalizada de los alumnos era que los mismos eran efectivamente las raíces, cuando previamente habían escrito que esos valores no formaban parte del dominio. Es decir que no evidenciaban la contradicción ni revisaban los resultados del software.

En cuanto a la habilidad Identificar continuidad en intervalo cerrado la dificultad que observamos es que los alumnos comprobaban la continuidad solo en los valores extremos del intervalo, pero no analizaron en los puntos interiores 
al intervalo.

\subsubsection{Actividades de construcción}

\section{Tema: Límite, continuidad y asíntotas}

Mostrar usando el Mathematica gráficos de funciones que satisfagan las condiciones dadas en cada caso (puede ser una sola por caso).

a) Una función que tenga dos asintotas oblicuas.

b) Una función que tenga límite finito en un punto y NO sea continua en el punto.

Esta es una actividad de construcción ya que el alumno debe inventar una función con cada una de las características solicitadas. La particularidad de este ejercicio radica en que al trabajar con el software, la construcción debe ser analítica para poder luego realizar el gráfico.

\section{Análisis descriptivo}

Las habilidades promovidas por la actividad son: Controlar - Explorar.

La vinculación que realizamos en este caso de cada habilidad en relación con el contenido es:

- Controlar asíntota oblicua.

- Explorar existencia dos asíntotas oblicuas.

- Controlar discontinuidad evitable.

- Explorar funciones con discontinuidad evitable.

Consideramos una calificación "Bien" (B) al alumno que:

- Brindó un ejemplo de una función con dos asíntotas oblicuas diferentes, y otra función con una (o más) discontinuidad evitable.

- En cuanto a la exploración, consideramos que el alumno exploró (Bien) cuando quedó plasmado en su producción impresa que buscó, indagó, graficó, probó con diferentes funciones, tratando de encontrar aquellas que cumplan con la consigna.

En el caso que alguno de estos aspectos no esté completamente bien, evaluamos con "Regular" (R) y si no responde o lo efectuado no es correcto, consideramos como calificación "Mal" (M).

La mayoría de los alumnos pudo controlar ambos conceptos y explorar con el software para encontrar funciones que cumplan con lo solicitado. En este caso fue de total importancia la facilidad que otorga la computadora para 


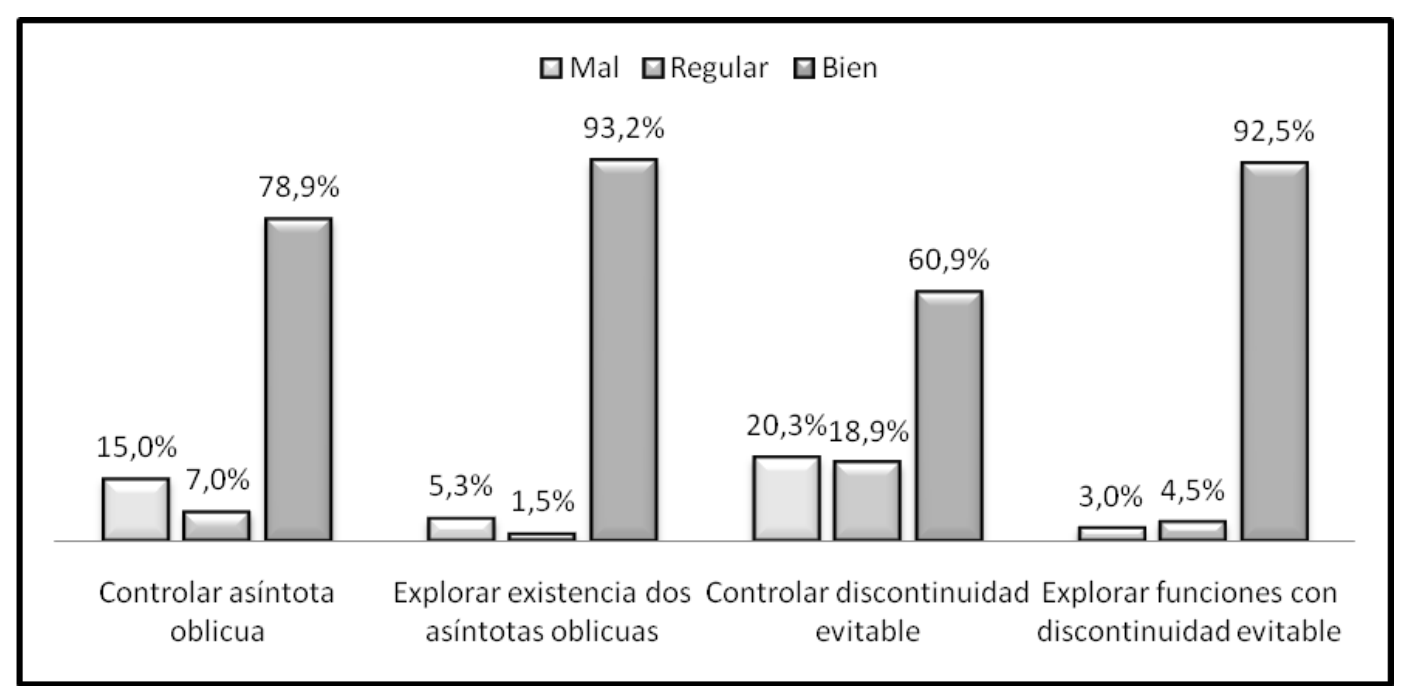

Figura 1.6 Gráfico de distribución de porcentajes de desempeño de cada habilidad-contenido

graficar y explorar distintas situaciones (NCTM, 2003).

\section{Análisis factorial de las variables}

Una vez realizado el análisis factorial, obtuvimos un agrupamiento en una sola componente con casi $50 \%$ de la varianza explicada.

\begin{tabular}{|l|l|}
\hline & Componente \\
\cline { 2 - 2 } & 1 \\
\hline Explorar existencia dos asíntotas oblicuas & 0,826 \\
\hline Controlar asíntota oblicua & 0,713 \\
\hline Explorar funciones con discontinuidad evitable & 0,739 \\
\hline Controlar discontinuidad evitable & 0,488 \\
\hline
\end{tabular}

Tabla 1.5

Aquí decidimos reducir las variables a una sola:

$$
y_{1}=0,826 x_{1}+0,713 x_{2}+0,739 x_{3}+0,488 x_{4}
$$

En este caso, no parece haber una desagregación por tema (asíntota oblicua, discontinuidad evitable), lo que no acuerda con lo que veníamos observando hasta ahora en todas las actividades. Una explicación posible de esto es el tipo de actividad constructiva propuesta, en la que deben responder con un objeto que cumpla condiciones, lo cual hace que las habilidades estén íntimamente relacionadas. En el otro tipo de actividades, en las que había más de una componente, se proponía ir respondiendo parcialmente distintos ítems.

\subsection{Conclusiones}

Al finalizar esta etapa del trabajo hemos podido diseñar distintos tipos de actividades y desarrollar una metodología para estudiar las diferentes habilidades que las mismas propician. 
Como resultado de este estudio extraemos las siguientes conclusiones que atraviesan todo el trabajo:

- Sobre las actividades y el desarrollo de habilidades matemáticas:

- Actividades de generalización y estudio de casos promueven habilidades como Explorar, Comparar, Generalizar y Fundamentar: el uso del software ayudó al desarrollo de la habilidad explorar y comparar debido a las facilidades de realizar gráficos, permitiendo visualizaciones rápidas y claras de las diferencias entre los gráficos de las funciones de acuerdo a los parámetros utilizados. La generalización y la fundamentación se han visto favorecidas pero en menor grado con respecto a las habilidades Explorar y Comparar.

- Actividades donde el alumno aplica conceptos estudiados en la teoría promueven habilidades como Identificar: esto estaría contribuyendo al afianzamiento de los conceptos, ya que el alumno al aplicarlo a otras situaciones puede identificar dicho concepto y usarlo.

- Actividades de aplicación de algoritmos propician Identificar, Analizar, Calcular y Controlar: esto podría deberse a que la aplicación de algoritmos siempre está contextualizada, siempre está aplicada a un problema particular, en el cual es necesario poner en práctica determinados conceptos teóricos. Por lo tanto, mediante este tipo de actividades estaríamos promoviendo la identificación, análisis y control de dichos conceptos. También favorece el cálculo lo que ayuda a una automatización de procedimientos

- Actividades de construcción favorecen el Explorar y Controlar: esto puede deberse a que el software ofrece facilidades de gráfico y el alumno puede dirigir su atención al concepto, ayudando así a una mayor exploración, lo que contribuye a un aumento de la habilidad controlar

- Sobre el uso del software en el diseño de las actividades.

- Aprovechamiento de características del software: las actividades propuestas aprovechan las posibilidades que ofrece el software, especialmente las de graficación, resolución de ecuaciones, cálculo numérico y cálculo de límites. Esto exige tanto a docentes como alumnos, un conocimiento sobre el software y, particularmente para los docentes, sobre cómo orientar a los alumnos en su uso.

- Potencialidad didáctica del uso del software: en todos los casos las habilidades estudiadas se vieron manifestadas aceptablemente. Consideramos que la visualización, el uso de gráficos, el ahorro de los cálculos simbólicos y numéricos que realiza el programa sin esfuerzo por parte del alumno, la exploración de varios casos, entre otros, han sido una razón para el desarrollo o fortalecimiento de tales habilidades.

- Sobre la enseñanza de la asignatura:

- En esta ocasión ya se habían dado los temas, por lo que el trabajo en el taller sirvió para repasar o para fortalecer los conceptos vistos en las actividades. Esto nos estaría remarcando la importancia de una instancia de taller con uso de software, paralelo al dictado de la asignatura, ya que es un espacio en el cual los alumnos pueden consultar y afianzar los contenidos. La propuesta de un tipo de trabajo distinto del de la clase incentivó a los estudiantes a consultar nuevamente los temas y a ver otro enfoque de los mismos.

- Sobre el análisis factorial de las habilidades desarrolladas:

- Dada la hipótesis de vinculación de las habilidades por contenido, esperábamos que el análisis factorial nos permitiera identificar grupos de habilidades. Vimos que en la mayoría de los casos éstas se agruparon por el tipo de tarea a realizar, por ejemplo cálculo de asíntotas, por un lado, determinación de dominio de la función por el otro. Además, en general, aparecieron no más de dos componentes, lo que estaría mostrando la estrecha relación entre los desarrollos de habilidades y la tarea realizada, dando cuenta de la efectividad de los ejercicios propuestos y del modo de trabajarlos según nuestra propuesta. Esto nos alienta además a pensar que es conveniente considerar una clasificación del tipo habilidad-contenido. 
- Una lectura del análisis descriptivo y factorial, nos hace notar algunas cuestiones sobre los presupuestos de enseñanza que a veces tenemos los docentes. Por ejemplo nos invita a reflexionar sobre el papel del "caso paradigmático". En las actividades sobre las transformaciones de los gráficos (ver análisis de la actividad 1 de generalización y de la actividad 3 de aplicación) esperábamos que los que hicieran bien la primera parte de la actividad pudieran, respectivamente, generalizar por analogía o controlar mediante comparación de gráficos la función inicial con la resultante de acuerdo a la consigna. Otro punto para la reflexión es el significado asignado por los estudiantes a ciertas tareas, por ejemplo en la actividad donde se les pedía el análisis de la continuidad de la función en un intervalo cerrado, la mayoría de los alumnos estudió solo la continuidad en los extremos y no en el interior. Probablemente esto sucedió porque los estudios de los casos "escolarizados" en los que se estudia la continuidad, como el caso de las funciones a trozos, en general no presentan dificultad de análisis en el interior del intervalo sino en los extremos.

\section{Bibliografía}

[1] Bronk R. “Bloom's Taxonomy”. En http://sites.wiki.ubc.ca/etec510/Bloom's_Taxonomy (consultada el 13 de Julio de 2011)

[2] Contreras De La Fuente A., Font Moll V., García Armenteros M., Luque Cañada L., Marcolini Bernardi M., Ordóñez Cañada L., Ortega Carpio M. y Sánchez Gómez C. "Aplicación del programa "Mathematica a las prácticas de Cálculo en el Primer Año Universitario". Noveno Simposio De La Sociedad Española De Educación Matemática SEIEM (2005). p 271-282.

[3] Consejo Federal de Decanos de Ingeniería (CONFEDI). “Primer acuerdo sobre competencias genéricas. Primer y segundo informe". En http://www.confedi.org.ar/component/option, com_docman/task, cat_view/gid, 20/ dir, DESC/order, date/limit, 5/limitstart, 5/ (consultada el 9 de septiembre de 2007)

[4] Cuicas Ávila, M., Debel Chourio, E., Casadei Carniel, L. y Álvarez Vargas, Z. “El software matemático como herramienta para el desarrollo de habilidades del pensamiento y mejoramiento del aprendizaje de las matemáticas". Actualidades Investigativas en Educación (2007) 7 (2), 1-34.

[5] Churches, A. “Taxonomía de Bloom para la era digital”. En http://www. eduteka.org/TaxonomiaBloomDigital. php (consultado el 1 de febrero de 2012)

[6] Elena, C. y Herrera, C. "El desarrollo de habilidades matemáticas en alumnos de primer año de Ingeniería". En http://www.famaf.unc.edu.ar/rev_edu/documents/vol_22/pro_Herrera_tra.pdf (consultada el 17 de febrero de 2009)

[7] Falsetti M., Favieri A., Scorzo R., and Williner B. "Estudio sobre habilidades matemáticas para el cálculo diferencial en estudiantes de ingeniería." Actas Del 10mo Simposio De Educación Matemática. (2009). p. $303-321$.

[8] Goldenberg P. Pensando y hablando de tecnología en la clase de matemática. En http://www.eduteka.org/ tema_mes.php3 (consultada el 10 de agosto de 2011).

[9] Gómez, M. Introducción a la metodología de la investigación científica. Editorial Brujas. 2006.

[10] Hernaìndez, Fernaìndez H., Delgado Rubí J. y Fernández De Alaíza, B. Cuestiones de Didaìctica de la Matemaìtica: Conceptos Y Procedimientos en la Educacioin Polimodal y Superior. Homo Sapiens. 1998.

[11] Hernández Rodríguez, O. Temas de análisis estadístico multivariado. Editorial Universitaria de Costa Rica, 1998. 1ra ed.

[12] Hitt, F. "Una reflexión sobre la construcción de conceptos matemáticos en ambientes con tecnología". Boletín de la Asociación Matemática Venezolana, 10 (2) (2003). En http://www.emis.de/journals/BAMV/conten/vol10/ fernandoHitt.pdf (consultada el 8 de agosto de 2011)

[13] Katz, S. y Cámara, V. “Las habilidades cognitivas y hábitos de estudio en matemática: un diseño posible para su investigación". En http://www.fpsico.unr.edu.ar/congreso/mesas/Mesa \{ㅇ 205/marciparkatzcamara.pdf (consultada el 10 de noviembre de 2010)

[14] Lasnier, F. Reússir la formation par compétences. Guérin. 2000. 
[15] Larson R., Hostetler, R., y Edwars, B. Cálculo I: Vol. 1. Ediciones Pirámide. 2002. 7ma ed.

[16] Luzón Encabo, J.M. "La informática desde la perspectiva de los educadores". Congreso Internacional de Informática Educativa 97. Vol 1. Universidad Nacional de Educación a Distancia, 1997.

[17] Macías Ferrer D. “Las nuevas tecnologías y le aprendizaje de las matemáticas”. Revista Iberoamericana de Educación (2007), 42 (4). http: / / www.rieoei.org/deloslectores/1517Macias.pdf (consultada el 10 de diciembre de 2010).

[18] Modarelli, M; Nolasco, M., Boucíguez, M., Irassar, L., Suárez, M y Berrino, M. “Resolución de problemas: una herramienta útil para desarrollar habilidades matemáticas". En http://online2.exactas.unlpam.edu.ar/repem/ cdrepem06/memorias/comunicaciones/Propuestas/CPD 4 .pdf (consultada el 3 de noviembre de 2010)

[19] Muñoz Carril, P. C. y González Sanmamed, M. “Conocimientos del profesorado universitario en herramientas telemáticas". CPU-e, Revista de Investigación Educativa, 13 (2011).

[20] NCTM. "Principios para matemáticas escolares. Consejo Estadounidense de Profesores de Matemáticas (NCTM)". En http: //www. eduteka.org/PrincipiosMath.php (consultado el 20 de agosto de 2011).

[21] Piskunov, N. Cálculo diferencial e integral. Editorial Mir. 1977. 3ra ed.

[22] Rodríguez, M., Carnelli, G. y Formica, A. “Una evaluación de habilidades matemáticas”. Suma (2005) $48,33-43$.

[23] Stewart, J. Cálculo Conceptos y contextos.: Thomson Editores. 1999.

[24] Yániz Álvarez, C. Planificar la enseñanza para el desarrollo de competencias. Educatio Siglo XXI (2006) 24,17-34. 